\title{
Ruptura del musculo gastronecmio en una novilla Gyr en el departamento de Córdoba, Colombia.
}

\section{Rupture of the gastrocnemius muscle in a heifer Gyr in the department of Córdoba, Colombia.}

\author{
Cardona A, José ${ }^{1 *}$ Ph.D, Suarez Ch, Alfredo ${ }^{1}$ M.Sc, Isaías A, Jimenez MVZ,
}

${ }^{1}$ Universidad de Córdoba, Departamento de Ciencias Pecuarias, Grupo de Investigaciones en Medicina de Grandes Animales (MEGA), Montería, Colombia.

\section{Key words:}

\section{Muscle;}

rupture;

bovine;

lameness;

(Source: MeSH).

\begin{abstract}
The case of a 1.5-year-old female Pure Gyr Bovine, which was attended by the outpatient clinic of the Medical-Surgical Clinic area of Large Animals of the Faculty of Veterinary Medicine and Zootechnics of the University of Cordoba. The animal was found in the recumbent paddock, following a low epidural anesthetic procedure for follicular aspiration purposes. He was incapable of joining with severe manifestations of pain. On standing he showed plantar support with the metatarsus of the left hind limb. General clinical examination and radiology of the affected area were performed. In the Clinical evaluation of the animal in right lateral decubitus, inflammation was found on the proximal and medial region of the tibia of the left hind limb, on palpation showed acute signs of pain and muscle tremors in the affected area as well as Pain and sagging at the level of the gastrognemius tendon, later the animal was stopped, showing plantar support in the season with the whole metatarsus. The definitive diagnosis was of rupture of the gastrognemius tendon.
\end{abstract}

\section{Resumen}

Músculo;

ruptura;

bovino;

cojera;

(Fuente: $\mathrm{MeSH}$ ).

INFORMACIÓN

Recibido: 22-02-2016;

Aceptado: 05-06-2016.

Correspondencia autor:

cardonalvarez@hotmail.com
Se expone el caso de un Bovino Gyr puro, Hembra, de 1,5 años de edad, la cual fue atendida por el servicio clínico ambulatorio del área de clínica de MédicoQuirúrgica de Grandes Animales de la Facultad de Medicina Veterinaria y Zootecnia de la Universidad de Córdoba. El animal fue encontrado en el potrero en decúbito, posterior a un procedimiento anestésico de epidural baja con fines de aspiración folicular. Presentó incapacidad para incorporarse con fuertes manifestaciones de dolor, al colocarse de pie mostró apoyo plantar con el metatarso del miembro posterior izquierdo, fue realizado examen clínico general y radiología del área afectada. En la evaluación Clínica del animal en decúbito lateral derecho, se encontró a la inspección inflamación en la región proximal y medial de la tibia del miembro posterior izquierdo, a la palpación mostró signos agudos de dolor y temblores músculo cutáneos en el área afectada, así como dolor y flacidez a nivel del tendón gastrognemio, posteriormente se procedió a parar al animal, mostrando en estación apoyo plantar con todo el metatarso. El diagnóstico definitivo fue de con ruptura del tendón gastrognemio. 


\section{INTRODUCCIÓN}

Gracias a su ubicación geográfica Colombia cuenta con gran variedad de pisos térmicos que van desde el nivel del mar hasta regiones de páramo, ello permite la explotación de diferentes razas bovinas productoras de carne, leche y doble propósito.

La Población Bovina en el país está distribuida en 494.402 predios y constituida aproximadamente por $22^{\prime} 689.420$ animales, ubicados principalmente en los departamentos de Antioquia $(11,67 \%)$, Córdoba $(8,61 \%)$, Casanare $(8,18 \%)$, Meta $(7,36 \%)$, Santander (6,26\%) Cesar (6,02\%), Caquetá $(5,94 \%)$, Cundinamarca $(5.57 \%)$ y Magdalena $(5,35 \%)$ que agrupan el $64,96 \%$ de la población total nacional. De acuerdo a su vocación productiva las razas se hacen más susceptibles a una u otra enfermedad, por lo que el análisis de su ubicación, manejo y desplazamiento resulta fundamental para el diseño de estrategias de prevención y control de enfermedades (ICA, 2016).

En los bovinos se pueden presentar rupturas del musculo gastrocnemio. Dicho musculo se extiende desde el tercio distal del fémur a la punta del corvejón. Surge mediante dos cabezas. Una cabeza lateral, desde la tuberosidad supracondilea lateral (borde la fosa supracondilea) y una cabeza medial, desde la tuberosidad supracondilea medial. La Inserción se da en parte plantar de la tuberosidad calcánea. La Acción extiende el corvejón y flexiona la articulación femorotibiorrotuliana; sin embargo, estas dos acciones no pueden hacerse de manera simultánea (MAGALHÃES et al. 2014).

La rotura del músculo-tendón del gastrocnemio es una enfermedad poco frecuente en el ganado Bovino, por lo general de ocurrencia unilateral. Las causas están relacionadas con un traumatismo directo, postración prolongada y el desequilibrio en aumento, lo que puede conducir a la sobrecarga repentina del miembro extendido por debajo del cuerpo, provocando la rotura (MAGALHÃES et al. 2014).

En el ganado vacuno, los sitios más comunes de rotura muscular están en la unión músculo-tendinosa, y en el origen o inserción del músculo, la ruptura del músculo gastrocnemio puede o sucede parcial o totalmente; En la ruptura parcial, Los signos son menos pronunciados, y el pronóstico es mejor que en la ruptura completa (ALTUĞ et al. 2007).

\section{MATERIALES Y MÉTODOS}

Una novilla de la Raza Gyr pura, de 1.5 años de edad y un peso aproximado de $180 \mathrm{Kg}$. Fue atendida por el servicio clínico ambulatorio del área de clínica de Médico-Quirúrgica de Grandes Animales de la Facultad de Medicina Veterinaria y Zootecnia de la Universidad de Córdoba.

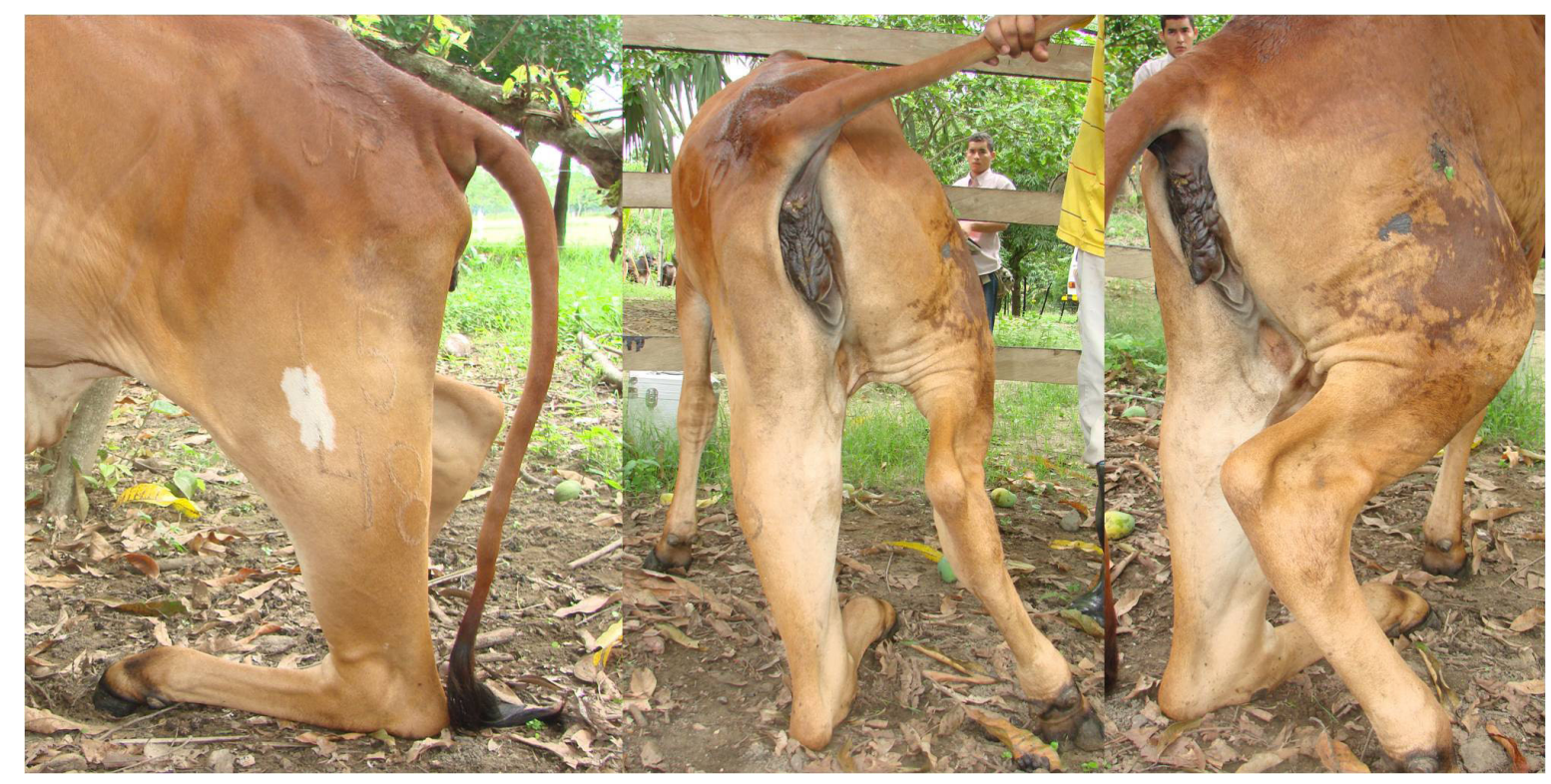

Figura 1. Apoyo plantar con el metatarso, obsérvese aumento de volumen en la región tibial en su cara lateral y posterior. 
Entre los datos anamnesicos, se tiene que la novilla fue encontrada en el potrero en decúbito, al siguiente día de haber sido sometida a un proceso de aspiración folicular con procedimiento anestésico de epidural baja. Se incitó a pararse mostrando manifestaciones de dolor y apoyo plantar con el metatarso del miembro posterior izquierdo, el servicio clínico fue solicitado 8 días posterior la alteración, por lo que fue solicitado la realización de pruebas radiológicas del área afectada.

En la evaluación Clínica del animal en decúbito lateral derecho, se encontró a la inspección inflamación en la región proximal y medial de la tibia del miembro posterior izquierdo, a la palpación mostró signos agudos de dolor y temblores músculo cutáneos en el área afectada, así como dolor y flacidez a nivel del tendón gastrognemio, posteriormente se procedió a parar al animal, mostrando en estación apoyo plantar con todo el metatarso, siendo compatibles todas estas manifestaciones clínicas con ruptura del tendón gastrognemio (Figura 1) y fisura de la tibia en el tercio proximal (Figura 2). En el resto de sus sistemas presentaba normofuncionalidad.

\section{RESULTADOS Y DISCUCCIONES.}

La ruptura del tendón gastrognemio se debió probablemente a un traumatismo causado por otro bovino o por apoyo inadecuado debido a la pérdida de propiocepción ocasionada por la anestesia epidural baja, resultados similares fueron reportados por ALTUĞ et al. (2007) y SIVARAMAN et al. (2012), dependiendo si la ruptura fuese total o parcial así será su pronóstico siendo en este medio un factor limitante un quirófano de grandes con la infraestructura necesaria para poder llevar a buen término un post-operatorio.

En la etiología de la ruptura del músculo gastrocnemio, se encuentran lesiones resultantes de situaciones de saltos y caídas, lo que causa una debilidad de los músculos, y los esfuerzos para levantarse terminan en la ruptura del mismo (JESTY et al. 2005; ALTUĞ et al. 2006; ALTUĞ et al. 2007).

Otra de las posibles causas en animales adultos de ruptura del músculo gastrocnemio puede ser en la mayoría de los casos, el proceso de monta inesperado por el toro o de otra vaca en el proceso de celo. SIVARAMAN et al. (2012), manifestaron que los animales afectados fueron incapaces de extender el corvejón, por lo que caminaban sobre sus corvejones en casos de ruptura total y algunos con flexión y caída del corvejón si la ruptura fue parcial.

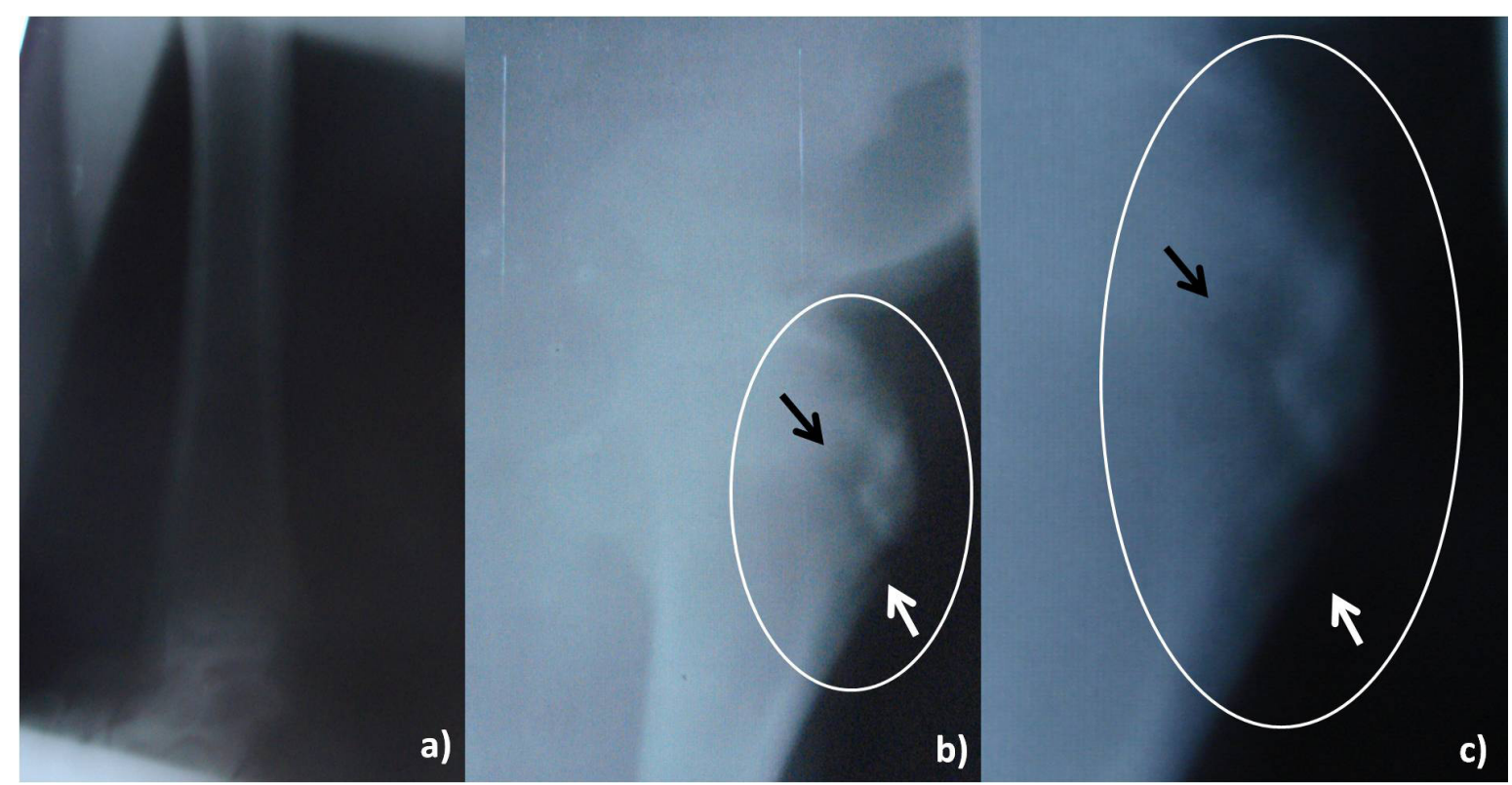

Figura 2. Tercio medio y distal de la tibia con apariencia radiológica normal (a), epífisis proximal de la tibia en su cara anterior, observe el área de la fisura encerrada en el círculo (b) $(\rightarrow)$, Aproximación de la fisura (c) $(\rightarrow)$. 


\section{REFERENCIAS}

ALTUĞ, N.; AĞAOĞLU, Z.; YÜKSEK, N.; KAYA, A.; KELEŞ, I. 2006. Adenosine deaminase in the diagnosis of white muscle diseases in lambs. Med Weter 62: 1007-1010.

ALTUĞ, N.; ÖZKAN, C.; YÜKSEK, N.; KARASU1, A.; KELEŞ, I.; AĞAOĞLU, Z.; ILHAN, F. 2007. Rupture of the gastrocnemius muscle in a cow two months after twin birth. Bull Vet Inst Pulawy 51: 615-619.

INSTITUTO COLOMBIANO AGROPECUARIO (ICA). Censo Pecuario Nacional - 2016. Disponible desde internet: http://www.ica.gov.co/getdoc/8232c0e5-be97-42bd-b07b-9cdbfb07fcac/Censos-2008.aspx.

JESTY, S.; PALMER, E.; PARENTE, E.; SCHAER, T.; WILKINS, P. 2005. Rupture of the gastrocnemius muscle in six foals. J Am Vet Med Assoc, 227: 1965-1968.

MAGALHÃES, L.; ANJOS, C.; NOGUEIRA, G.; SAUT, J. 2014. Evolução clínica de ruptura de gastrocnêmio em bovino da Raça Nelore. Anais do XI Congresso Brasileiro de Cirurgia do CBACV e I Congresso Internacional de Cirurgia do CBCAV. Pag. 55.

SIVARAMAN, S.; VENKATESAKUMAR, E.; VIJAYAKUMAR, G.; KUMARESAN, A., SUBRAMANIAN, M. 2012. Gastrocnemius muscle ruptur in a cow. A case report. JIVA. 10 (2): 55 - 56. 